\title{
Association of Drusen Deposition with Choroidal Intercapillary Pillars in the Aging Human Eye
}

\author{
Imre Lengyel, ${ }^{1,2}$ Adnan Tufail, ${ }^{1}$ Heba Al Hosaini, ${ }^{1}$ Pbilip Lutbert, ${ }^{1}$ Alan C. Bird, ${ }^{1}$ and \\ Glen Jeffery ${ }^{1}$
}

Purpose. To determine the pattern of drusen accumulation with age and to investigate the initial sites of deposition and their relationship to choroidal capillaries in human donor eyes from the eye bank of Moorfields Eye Hospital.

Methods. Wholemounted, hydrated preparations of the choriocapillaris and Bruch's membrane from donor eyes ranging from 42 to 95 years, with or without retinal pigment epithelium (RPE), were examined by conventional and confocal microscopy. Drusen were visualized by their autofluorescence.

RESuLTs. In all age groups studied autofluorescent drusen were present at the equator but were not found centrally where the vascular architecture is different, being tubular rather than a honeycomb pattern. Autofluorescing drusen were strongly associated with the lateral walls of the choriocapillaris (an area commonly known as the intercapillary pillars of the choriocapillaris $(P=0.028$; Wilcoxon signed ranks test $)$. Nonfluorescing drusen were occasionally seen centrally, but were not easily identified, and because of their large size, their localization with respect to capillary walls was not possible.

Conclusions. These results strongly support the notion that autofluorescent drusen are not randomly distributed and have a specific spatial relationship to choroidal vessel walls. That equatorial drusen fluoresce, whereas central drusen do not, suggests that they may have different chemical compositions at the two sites and possibly different significance in age-related macular disease. (Invest Ophthalmol Vis Sci. 2004;45: 2886-2892) DOI:10.1167/iovs.03-1083

$\mathrm{D}$ rusen are focal deposits that accumulate between the retinal pigment epithelium basal membrane and the inner collagenous layer of Bruch's membrane. ${ }^{1,2}$ The size, number, and/or degree of confluence of drusen are associated with aging and are risk factors for the development of age-related macular degeneration (AMD). ${ }^{1,3-6}$ Although the pathogenesis of drusen has not been fully elucidated, various investigators have proposed that the accumulation of abnormal material between the outer retina and the choriocapillaris induces RPE and/or neural retinal damage by interfering with the exchange of nutrients and waste products and/or by inhibiting bulk fluid flow. ${ }^{7,8}$

From the ${ }^{1}$ Experimental Age Related Macular Degeneration Research Group, Institute of Ophthalmology, University College London, London, United Kingdom; and the ${ }^{2}$ Department of Biochemistry, Biological Research Centre, Szeged, Hungary.

Supported by Wellcome Trust (GJ), the Hungarian Research Fund (T037911), and the Mercer Fund (IL).

Submitted for publication October 1, 2003; revised February 4 and April 8, 2004; accepted April 22, 2004.

Disclosure: I. Lengyel, None; A. Tufail, None; H. Al Hosaini, None; P. Luthert, None; A.C. Bird, None; G. Jeffery, None

The publication costs of this article were defrayed in part by page charge payment. This article must therefore be marked "advertisement" in accordance with 18 U.S.C. $\$ 1734$ solely to indicate this fact

Corresponding author: Glen Jeffery, Department of Visual Science, Institute of Ophthalmology, University College London, Bath St., London EC1V 9EL; g.jeffery@ucl.ac.uk.
The clinical, histopathological, and molecular heterogeneity of drusen are of interest in understanding the pathogenesis of AMD. Previous investigators have suggested that drusen are derived from a variety of sources, including the retina and choroid, and that they contain proteins, ${ }^{9-16}$ lipids, ${ }^{17-20}$ and trace elements. ${ }^{21-24}$ The diversity of these constituents was confirmed by a recent proteome analysis. ${ }^{25}$ Freidman et al. ${ }^{26}$ have proposed that there is a relationship between drusen deposition and the choriocapillaris. However, this relationship has not been explored quantitatively in whole eyes of various ages. In the present study, we investigated the initial sites where deposition occurs and the progression in such patterns with age, using the autofluorescent properties of drusen.

\section{MeTHODS}

\section{Subjects}

Human donor eyes with no known disease from donors aged between 42 and 95 years were obtained from the Eye Bank at Moorfields Eye Hospital. Full Local Research Ethics Committee approval and appropriate consent were obtained in each case. The protocol of the study adhered to the tenets of the Declaration for Helsinki regarding research involving human tissue.

\section{Tissue Preparation}

Forty-six eyes were received 24 to 50 hours after death with the corneas removed. Retinal tissue from similar ages has been analyzed in fully hydrated specimens and the integrity of individual cells examined. No significant postmortem artifacts were seen in the morphology. Cellular layers were clearly present. Within these layers, individual cells were identified with no obvious cellular debris (data not shown). The tissue was processed in one of two ways. The first group of eyes $(n=24)$ had the lenses removed and then placed in $10 \%$ buffered formalin. After fixation, the retina and attached RPE and choriocapillaris were dissected from the sclera. The neural retina was then separated from the RPE-choroid complex and the latter tissue wholemounted on a glass slide with the RPE facing upward. Excess liquid was removed, and the tissue was either viewed and photographed, or it was mounted in glycerol and coverslipped.

The second group ( $n=12$ ) were fellow eyes of the first group. These were stored in phosphate-buffered saline (PBS) until the RPE cells detached from the underlying basement membrane. The neural retina was then removed, and the remaining tissue washed in PBS to remove detached RPE cells and debris. The remaining choroid including Bruch's membrane, was then fixed, removed from the sclera, and mounted as just described. Drusen adhered firmly to Bruch's membrane and did not detach with the RPE, even after brushing and vigorous agitation in PBS.

Components of drusen have been shown to autofluoresce. ${ }^{27}$ In this study drusen distribution was determined by using $\lambda_{\text {ex }}$ of 460 to 500 $\mathrm{nm}$. This autofluorescent characteristic of drusen allowed visualization of both drusen and the choroidal capillary walls in the hydrated wholemounts of the retina. Structural details of these preparations were further elucidated by viewing the slide preparation with a confocal microscope (model LSM50; Carl Zeiss Meditec, Dublin, CA), using $\lambda_{\text {ex }}$ of $488 \mathrm{~nm}$ (laser power, 5\%; detector gain, 1074; amplification 
offset, 0.1655; and amplification gain, 1) and the emitted light was filtered by 530 to $560 \mathrm{~nm}$ cutoff filter. For the purpose of comparison with conventionally sectioned ocular tissue, sections of human aged eyes were obtained from the Department of Pathology of the Institute of Ophthalmology. These were paraffin-embedded and sectioned at 5 $\mu \mathrm{m}$, stained with hematoxylin-eosin and viewed with bright-field and $\lambda_{\text {ex }}$ of 460 to 500-nm fluorescent light. Their postmortem times were similar to donor eyes.

To compare drusen appearance in wholemounts with sectioned specimens, a 71-year-old donor eye was analyzed as a wholemount and then the central region was dissected, embedded in agarose, and frozen-sectioned transversely at $35 \mu \mathrm{m}$. These were viewed as just described.

\section{Tissue Analysis}

Digital images were acquired using a stereo zoom microscope with fluorescence attachment and (model DXM1200; Nikon) camera, and saved as TIFF (tagged information format) files. Each image was processed based on a previously published protocol ${ }^{28}$ using image-analysis software (Photoshop ver. 6.5; Adobe Systems, Mountain View, CA). In short, the total area occupied by the green fluorescing choroidal intercapillary pillars and drusen was determined using the "histogram" function, and the highlighted area was blue. In a second step, drusen were highlighted independently in a semiautomated fashion, using the "color range" function and the "eyedropper" tool to sample them. The "fuzziness" function was defaulted to $43 \%$. The "eyedropper" was then used to select a typical druse. The software at these settings isolated drusen from the capillary pillars based on their different shade of green. Additional drusen that were out of focus were selected manually using the "magic wand" tool (tolerance set to 15). The highlighted drusen were then changed to red using the "fill" command. These false-colored pictures then were exported into UTHSCSA Image Tool ver. 3.0 (http://ddsdx.uthscsa.edu/dig/itdesc.html/; provided in the public domain by the University of Texas Health Sciences Center San Antonio, TX), for numerical analysis. The size, number, and distribution of drusen were analyzed in a minimum of eight $2.6 \times 2.1-\mathrm{mm}$ (corresponding to 1280-1024 pixels) fields from each retinal quadrant from 42-, 66-, and 71-year-old donor eyes. Drusen position relative to the choroidal capillaries was determined manually by overlaying a grid on top of each highlighted drusen and determining whether they were on or off the intercapillary pillars or in an indeterminate position relative to the pillars. The area occupied by capillary lumen only was determined by subtracting the area of the blue pillars and drusen from the total field area. Then the ratio of the area occupied by the red drusen found between or on the choroidal capillary walls to those found elsewhere was determined. If drusen distribution was random, then one would expect drusen to be distributed on and off the pillars on average in the same ratio as the area of the pillars to the nonpillar area. All data were exported to a statistical analysis program (SPSS ver. 9.0; SPSS, Chicago, IL). The normality of the ratio distribution was determined using the Shapiro-Wilk Test. The ratios were compared using the Wilcoxon signed ranks test.

Maps of the relative location of drusen in complete wholemounts were made under the same microscope. Drawings of the wholemounts were made by outlining them on a large coverslip. The locations of drusen were marked directly on the overlaying coverslip with finetipped markers with the aid of retinal landmarks. No attempt was made to provide a detailed map with each druse marked. Rather the relative density was mapped onto these representations.

\section{Results}

First, preparations from donor eyes of ages spanning more than 50 years were viewed with the RPE in position. Characteristic images from one of the preparation (from a 71-year-old donor) can be seen in Figure 1. Figure 1A shows a region between the optic nerve head and the fovea, which appeared to be drusen free. The yellow-brown fluorescence represents the RPE, which is continuous across this region. However, in Figure 1D, which was taken from the equatorial region, numerous greenfluorescing drusen of different sizes were identified rupturing the RPE. Figure $1 G$ is a lower-magnification view from the equatorial region showing individual RPE cells and several large $(>200 \mu \mathrm{m})$ drusen that were formed by coalescing smaller $(10-50 \mu \mathrm{m})$ drusen. Where the RPE layer appeared discontinuous, pigment granules could be seen on top of individual druse and RPE cells around them were often distorted (Figs. 1D, 1G). The overall picture conveyed was one in which the physical integrity of localized regions of the RPE was compromised by drusen in a relatively random way in equatorial retinal regions.

Removal of the RPE revealed the vascular patterns of connective tissue between blood vessels, which fluoresced under the same excitation wavelength as drusen, but both remained readily distinguishable from one another. This was due not only to their different shapes, but also because they had slightly different emission wavelengths. In the central region, the pattern corresponded to the tightly packed structure of the choriocapillaris at this location ${ }^{29}$ (Fig. 1B). Although this honeycomb pattern was always present in the area between the optic nerve head and the fovea, its peripheral boundary was hard to define, and so we cannot relate it to classic anatomic terms, such as the macula or posterior pole. As with the preparations retaining the RPE (Fig. 1A), no green-fluorescing drusen were identified in this area. However, very rarely, druselike objects were seen, but only when the tissue was partially folded and pigment particles were present on their surface, highlighting their position. These were relatively large $(\sim 100 \mu \mathrm{m})$, translucent, dome-shaped objects that did not fluoresce (Fig. 1C). In equatorial regions, an extensive distribution of autofluorescing drusen could be seen after the RPE had been removed (Figs. $1 \mathrm{E}-\mathrm{M})$. Drusen had an irregular dome-shaped appearance, and were anchored to Bruch's membrane at their base (Figs. 1F, 1J). As in Fig. 1G, the larger ones often appeared to be formed by the coalescence of small drusen (Fig. 1H). A confocal crosssectional image of one of these drusen revealed the vacuolar internal structure (Fig. 1I) that is characteristic of some drusen. ${ }^{12,30}$

There was a striking spatial association between autofluorescing drusen and the choriocapillaris (Figs. 1E-M). As was clear when viewed in a horizontal cross section, autofluorescing drusen were consistently located internal to the intercapillary pillars of the choriocapillaris (Fig. 1J). In the equatorial region, the capillaries were well defined, appearing as long vessels with drusen highlighting the pillars (Figs. 1E, 1H, 1L, $1 \mathrm{M})$. In the transition zone, where there is a change in vascular pattern from a tubular to honeycomb-like configuration, ${ }^{31}$ drusen deposition occurred between the "cobblestones" formed by the anastomosing capillary walls, but not over the surface covering the vessel lumen (Fig. 1K). The numerical density of drusen varied from sparse (Fig. 1L) to abundant (Fig. $1 \mathrm{M})$. The observation that drusen deposition colocalizes with the pillars was confirmed statistically (Wilcoxon signed ranks test, $P=0.028$ ). In each of the eight fields of view examined from five donor eyes, the mean percentage of drusen that localized to the capillary pillars was $90.2 \%$, whereas only $0.9 \%$ were not located on pillars. The remaining $8.8 \%$ had an undetermined position. Mainly large coalesced drusen belonged to this group, which spanned several capillaries. Hence, their exact localization to a pillar was not possible. As the nature of semiautomated image analysis techniques is criterion dependent, differential interference (DIC) optics were used to verify the location of drusen.

The dome-shaped, nonfluorescing druselike deposits in the central region (Fig. 1C) were seen only in wholemount prep- 

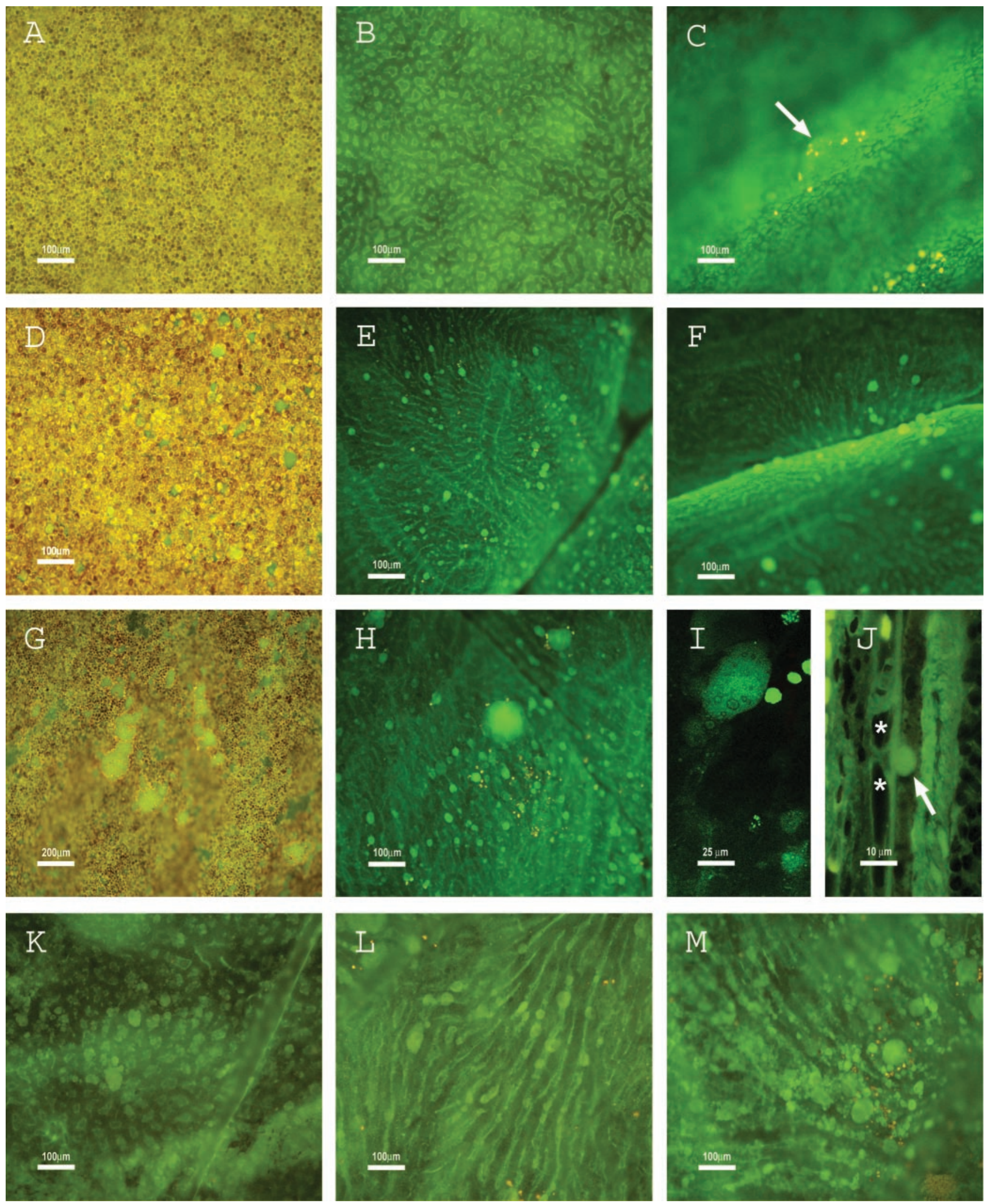

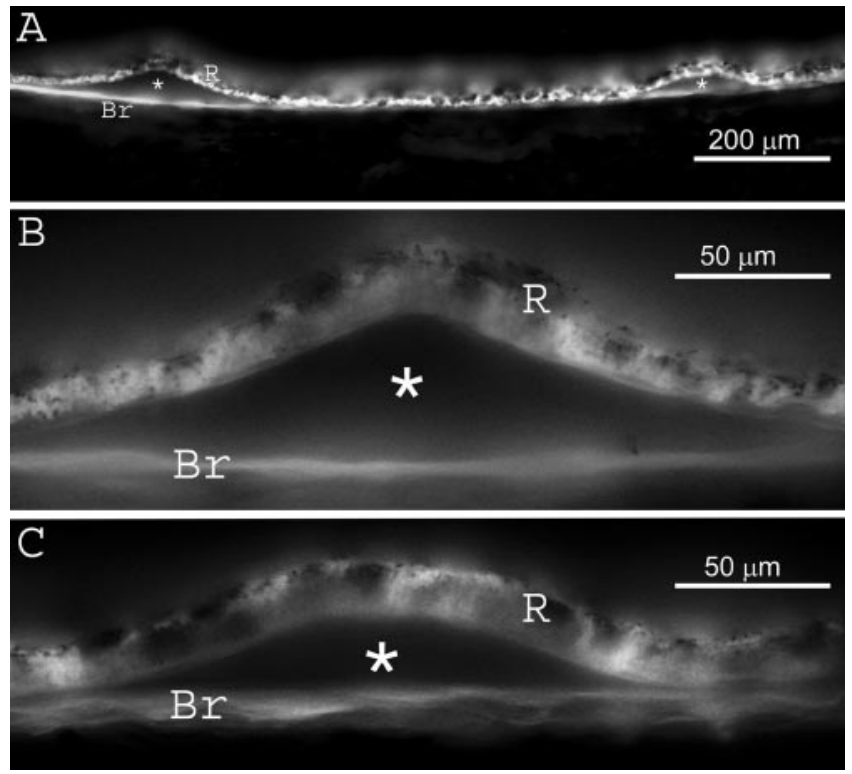

Figure 2. Transverse section of the central region of a 71-year-old donor eye. (A) Low-power micrograph in the central region viewed with fluorescence. Two distinct nonfluorescing drusen $(*)$ were apparent to the left and right, between the RPE (R) and Bruch's membrane $(\mathrm{Br})$. $(\mathbf{B}, \mathbf{C})$ Higher magnification of the left $(\mathbf{B})$ and the right $(\mathbf{C})$ drusen. In both cases the RPE was continuous across the surface of the druse. No fluorescence was apparent between the RPE and the Bruch's membrane.

arations when the RPE was removed. Therefore, the central region shown in Figure 1A was dissected and then frozen after agarose embedding, sectioned, and examined (Fig. 2). Discrete, shallow dome-shaped structures were apparent in these sections separating the RPE and Bruch's membrane. These did not fluoresce (Fig. 2) and did not show any internal structure when viewed by DIC optics (data not shown). Further, unlike the equatorial drusen, they did not rupture through the RPE.

In all preparations examined with the RPE present, autofluorescing drusen accumulation occurred in the equatorial region and was absent in more central areas such as the area between the fovea and the optic nerve head. Figure 3 shows a composite of wholemounted preparations from selected, representative individuals ranging from 42 to 95 years of age. The relative location of drusen is represented by dots. The location of the fovea is shown by an asterisk. Although an age-related increase in the number and the area occupied by drusen was observed, there was considerable variation in such features in donors of a similar age. There was a gradual increase in drusen and the area they occupied in tissues from the 42-, 71-, and 95-year-old donor eyes, with an annulus expanding in both directions from the equatorial region. However, exceptions were observed when these were compared with eyes from the 65- and 94-year-old donors. The preparation from a
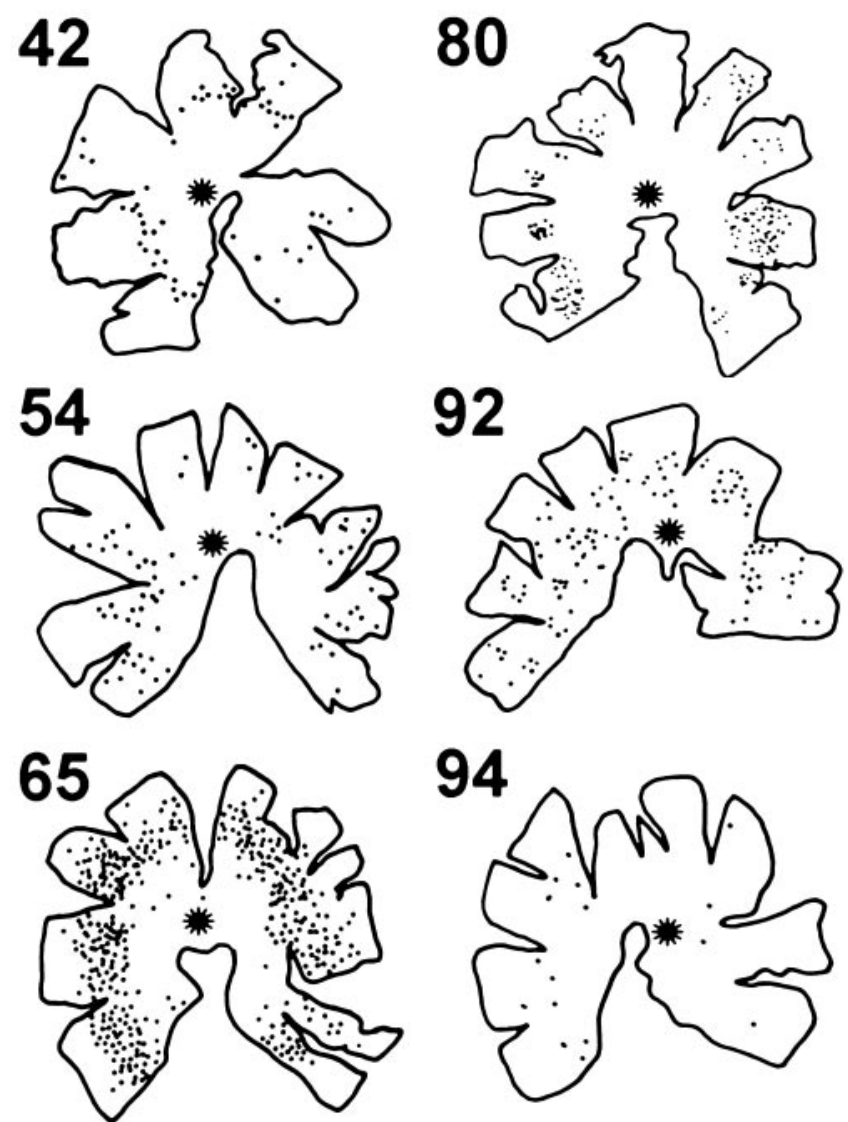

94

71

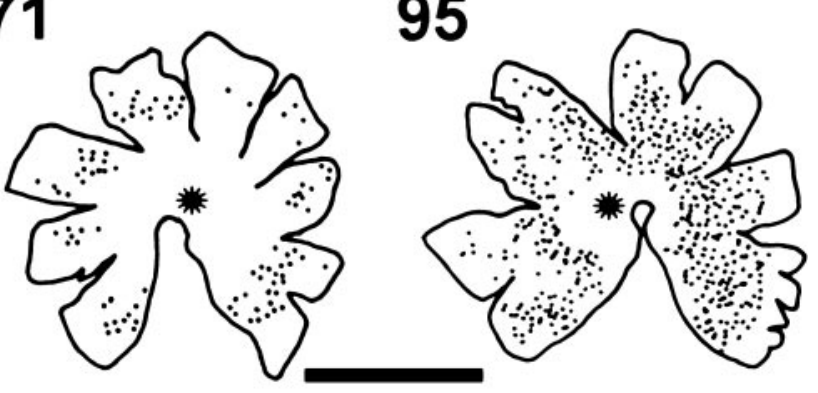

FIGURE 3. Distribution of drusen with RPE on at different ages (42-95 years). The relative density of the dots in each drawing represents the number and distribution of drusen. (**), location of the fovea. Although there was an increase in the number of drusen with age, exceptions were found (e.g., 94-year-old eye). Scale bar, $2 \mathrm{~cm}$.

65-year-old donor contained a relatively large number of drusen distributed over a wide area, whereas that from the 94-year-old eye contained markedly less drusen than any other eyes (Fig. 3). A comparison between the 94- and the 95-year-old donor preparations is particularly striking, as the former con-

FIGURE 1. Representative autofluorescence images of wholemounted wet preparations used in this study. Images from the central region of a 71-year-old donor eye with the RPE present (A) and with the RPE removed (B) revealing the underlying vascular pattern. A large druselike, nonfluorescing, domelike structure (arrow) was seen in this region when it was viewed in profile with yellow-fluorescing pigment particles attached to the surface (C). Representative images from the same eye, but from the equatorial region, showed drusen accumulation with the RPE present (D) and after its removal (E, F). Many small lens/dome-shaped drusen were identifiable in partial profile (F). Larger drusen were also present at the equatorial region when viewed with RPE on $(\mathbf{G})$ or off $(\mathbf{H})$. Confocal cross section of a druse revealed a vacuolar internal structure (I) characteristic of drusen. A paraffin-embedded section from the equatorial region showed a druse (arrow) located between two capillaries (*) and above the pillar separating them $(\mathbf{J})$. At the interface of the central region and the equatorial region drusen were present on the lateral walls of the choriocapillaries (K). There was a clear spatial association between drusen and the lateral walls of tubular choriocapillaries (L). Several areas showed very heavy drusen deposition (M). 


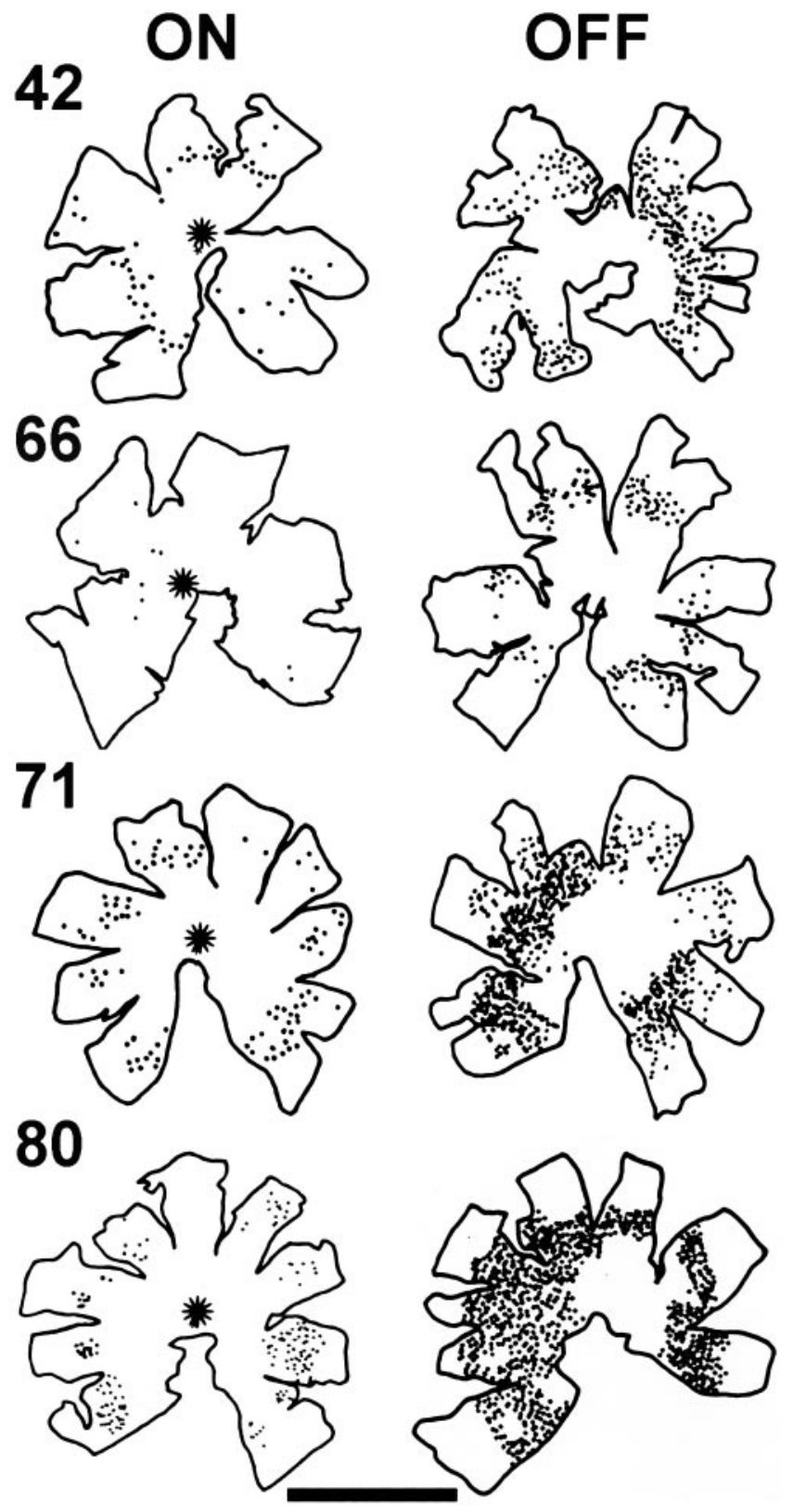

FIGURE 4. Distribution of drusen in fellow eyes with RPE on and off Considerably more drusen were identified when the RPE was removed

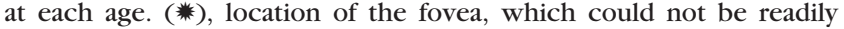
identified after RPE removal. Scale bar, $2 \mathrm{~cm}$.

tains very few drusen, whereas the latter contains a large number over an extensive geographic region. After the removal of RPE, an even more extensive distribution of drusen can be seen (Fig. 1). This is highlighted by a direct comparison of fellow eyes (Fig. 4) where the RPE was retained (ON) or removed (OFF). In each case, irrespective of the number of the drusen seen with RPE on, many more drusen were found after the removal of RPE, and they were distributed over a wider geographic region. The increase in the number of apparent drusen in the absence of RPE was most noticeable among the drusen of small size $(<10 \mu \mathrm{m})$, which, when the RPE was present, presumably had not yet extended through it. As seen with the RPE cells in place, the number of drusen increased with age (compare 42, 71, and 80); however, again, in the 66-year-old donor eye the number of drusen is lower than at any other age (Fig. 4).

\section{Discussion}

Druse formation is commonly associated with aging, 1,12,32,33 and it is widely accepted that with the progression of age, the number, size, and geographic extent of drusen increases in the macula. . $^{3,7,34-36}$ In our study, all donor eyes contained autofluorescing drusen, and there was an increasing number of these drusen with age, but there were exceptions to this. When the number of fluorescing drusen increased, there appeared to be an expansion of the band of highest density, both centrally, and peripherally. Equatorial drusen deposition colocalized with the intercapillary pillars of the choroidal capillaries. However, in no case did fluorescing drusen reach the far periphery. Autofluorescing drusen were also absent in the central area, characterized by the honeycomb pattern of choroidal capillaries. ${ }^{31}$

This raises the question of why we did not find autofluorescing drusen in the central region. Drusen are commonly seen clinically in the perifoveal region and we would have expected them to be present centrally in at least some of the aged eyes. ${ }^{3}$ Our inability to find fluorescing drusen in this region was the reason that we removed the RPE. Although removing the RPE exposed an extensive honeycombed vasculature centrally, still no fluorescing drusen were found. In a few cases we identified nonfluorescing elements with morphologic features similar to those described in more peripheral regions, ${ }^{37}$ and similar structures have recently been identified in the macular region (Hageman, personal communication, 2003). These were difficult to identify in wholemounts and were only seen in profile, assisted by the presence of RPE debris on their surface and the underlying autofluorescing capillary walls. Here, they appeared to have a different morphology than did the fluorescing drusen found at the equator. Similar nonfluorescing deposits were seen in hydrated sectioned tissue. Although these could be viewed as local retinal detachments, their appearance and location were strikingly similar to that in wholemounts with the RPE removed. However, a molecular approach would be needed to confirm clear differences between fluorescing and nonfluorescing deposits.

An examination of conventional paraffin-embedded sections from 40 randomly selected human donor eyes (age range, 22-95 years) stained with hematoxylin and eosin, with both fluorescence and light microscopy again failed to reveal autofluorescing drusen in the central region, although numerous equatorial drusen were found (data not shown). Furthermore, it has been shown that little autofluorescence can be detected from macular drusen seen clinically using the same excitation wavelength as we used. ${ }^{38}$ These add weight to the argument that drusen at the two locations may have a different chemical composition.

Distinctions between macular and peripheral drusen have already been made based on different lipid content, ${ }^{39}$ and it is possible that the process of dehydration associated with wax embedding and hematoxylin staining reduces their apparent number. Malek et al. ${ }^{39}$ also found that macular drusen are relatively hard to identify and, as such, called them "fragile." Drusen can be divided into several subclasses, ${ }^{34,37,40}$ and the ones we, and others, identified centrally could be a specific subclass. Proteomic analysis of drusen has produced valuable, but highly variable results, ${ }^{25}$ but no study has yet addressed whether or not the molecular content of drusen harvested from central and peripheral regions are different. Such data may be of key significance in determining the relationship between drusen at the two locations. Central drusen are clearly associated with sight-threatening complications of AMD, but determining the risks associated with equatorial drusen requires further clinical studies. 
An obvious distinction between the central and equatorial regions is their distinctive vascular patterns. ${ }^{31}$ In the central region there is an anastomotic capillary network supplying the densely packed photoreceptors in the adjacent neural retina. More peripherally, this pattern is less dense, and the vessels appear to be arranged longitudinally. The vortex vessels could also be readily identified in this area. Such differences in vascular pattern between the central and equatorial regions may be related to druse accumulation. The observation that druse distribution might be associated with the choroidal capillaries was suggested as early as 1856 by Müller, who provided the first description of the distribution of senile drusen. ${ }^{41}$ Subsequently, Friedman et al. ${ }^{26}$ also described a spatial relationship between drusen and choroidal capillaries in the periphery, similar to our own observations, but in dehydrated, stained specimens. However, this was only undertaken in limited regions of the retina, and the dehydration of the tissue produced shrinkage that closed the spatial relationship between adjacent blood vessels, limiting detailed analysis. Furthermore, no quantitative analysis was offered, nor were regional differences in choroidal capillary architecture ${ }^{31}$ appreciated. Unstained, hydrated wholemounts used here afford the closest approximation to the in vivo situation. Although no specific histologic stain is available for drusen, their fluorescence properties have been used both clinically ${ }^{42}$ and experimentally. ${ }^{27}$ Herein, we demonstrate quantitatively a very tight spatial relationship between drusen and the intercapillary spaces across the whole equatorial region at different ages.

There are a number of reasons why drusen may accumulate between capillaries. The most obvious is related to the clearance of cellular debris from the retina. ${ }^{12}$ As no luminal surface at the point of accumulation is present, debris cannot be cleared so readily. It is also possible that there may be differential unidentified adhesion characteristics in this region. It has been proposed that dendritic cells may play a role in this mechanism, as they can be recruited between blood vessels and act as a focus for debris originating in the RPE. ${ }^{37}$ Our results cannot distinguish between these, or other ${ }^{12,43}$ potential mechanisms. Nevertheless, our results clearly highlight the heterogeneity of druse subtypes and their respective geography, which is associated with marked differences in the architecture of choroidal capillaries.

\section{Acknowledgments}

The authors thank Marc Tibber and Peter Munro for help with confocal microscopy and Tünde Petó for comments on the manuscript.

\section{References}

1. Pauleikhoff D, Barondes MJ, Minassian D, Chisholm IH, Bird AC. Drusen as risk factors in age-related macular disease. Am J Ophthalmol. 1990;109:38-43.

2. Russell SR, Mullins RF, Schneider BL, Hageman GS. Location, substructure, and composition of basal laminar drusen compared with drusen associated with aging and age-related macular degeneration. Am J Ophthalmol. 2000;129:205-214.

3. Coffey AJ, Brownstein S. The prevalence of macular drusen in postmortem eyes. Am J Ophthalmol. 1986;102:164-171.

4. Lewis H, Straatsma BR, Foos RY. The prevalence of macular drusen in postmortem eyes. Am J Ophthalmol. 1986;102:801-803

5. Bressler SB, Maguire MG, Bressler NM, Fine SL. Relationship of drusen and abnormalities of the retinal pigment epithelium to the prognosis of neovascular macular degeneration. The Macular Photocoagulation Study Group. Arch Ophthalmol. 1990;108:14421447.

6. Holz FG, Wolfensberger TJ, Piguet B, et al. Bilateral macular drusen in age-related macular degeneration: prognosis and risk factors. Ophthalmology. 1994;101:1522-1528.
7. Pauleikhoff D, Harper CA, Marshall J, Bird AC. Aging changes in Bruch's membrane: a histochemical and morphologic study. Ophthalmology. 1990;97:171-178.

8. Bird AC. Bruch's membrane change with age. Br J Ophthalmol. 1992;76:166-168.

9. Hageman GS, Mullins RF, Russell SR, Johnson LV, Anderson DH. Vitronectin is a constituent of ocular drusen and the vitronectin gene is expressed in human retinal pigmented epithelial cells. FASEB J. 1999;13:477-484.

10. Anderson DH, Hageman GS, Mullins RF, et al. Vitronectin gene expression in the adult human retina. Invest Ophthalmol Vis Sci. 1999;40:3305-3315.

11. Anderson DH, Ozaki S, Nealon M, et al. Local cellular sources of apolipoprotein $\mathrm{E}$ in the human retina and retinal pigmented epithelium: implications for the process of drusen formation. $\mathrm{AmJ}$ Ophthalmol. 2001;131:767-781.

12. Hageman GS, Luthert PJ, Chong VNH, et al. An integrated hypothesis that considers drusen as biomarkers of immune-mediated processes at the RPE-Bruch's membrane interface in aging and age-related macular degeneration. Prog Retin Eye Res. 2001;20: 705-732.

13. Anderson DH, Mullins RF, Hageman GS, Johnson LV. A role for local inflammation in the formation of drusen in the aging eye. Am J Ophthalmol. 2002;134:411-431.

14. Johnson LV, Leitner WP, Rivest AJ, et al. The Alzheimer's A betapeptide is deposited at sites of complement activation in pathologic deposits associated with aging and age-related macular degeneration. Proc Natl Acad Sci USA. 2002;99:11830-11835.

15. Johnson PT, Lewis GP, Talaga KC, et al. Drusen-associated degeneration in the retina. Invest Ophthalmol Vis Sci. 2003;44:44814488.

16. Johnson LV, Leitner WP, Staples MK, Anderson DH. Complement activation and inflammatory processes in Drusen formation and age related macular degeneration. Exp Eye Res. 2001;73:887-896.

17. Mullins RF, Hageman GS. Human ocular drusen possess novel core domains with a distinct carbohydrate composition. J Histochem Cytochem. 1999; 47:1533-1540.

18. Curcio CA, Millican CL, Bailey T, Kruth HS. Accumulation of cholesterol with age in human Bruch's membrane. Invest Ophthalmol Vis Sci. 2001;42:265-274.

19. Farkas TG, Sylvester V, Archer D, Altona M. The histochemistry of drusen. Am J Ophthalmol. 1971;71:1206-1215.

20. Haimovici R, Gantz DL, Rumelt S, Freddo TF, Small DM. The lipid composition of drusen, Bruch's membrane, and sclera by hot stage polarizing light microscopy. Invest Ophthalmol Vis Sci. 2001;42: 1592-1599.

21. van der Schaft TL, de Bruijn WC, Mooy CM, Ketelaars DA, de Jong PT. Element analysis of the early stages of age-related macular degeneration. Arch Ophthalmol. 1992;110:389-394.

22. Ulshafer RJ, Allen CB, Nicolaissen B Jr, Rubin ML. Scanning electron microscopy of human drusen. Invest Ophthalmol Vis Sci. 1987;28:683-689.

23. Olin KL, Morse LS, Murphy C, et al. Trace element status and free radical defense in elderly rhesus macaques (Macaca mulatta) with macular drusen. Proc Soc Exp Biol Med. 1995;208:370 -377.

24. Hahn P, Milam AH, Dunaief JL. Maculas affected by age-related macular degeneration contain increased chelatable iron in the retinal pigment epithelium and Bruch's membrane. Arch Ophthalmol. 2003;121:1099-1105.

25. Crabb JW, Miyagi M, Gu X, et al. Drusen proteome analysis: an approach to the etiology of age-related macular degeneration. Proc Natl Acad Sci USA. 2002;99:14682-14687.

26. Friedman E, Smith TR, Kuwabara T. Senile choroidal patterns and drusen. Arch Ophthalmol. 1963;69:114-124.

27. Marmorstein AD, Marmorstein LY, Sakaguchi H, Hollyfield JG. Spectral profiling of autofluorescence associated with lipofuscin, Bruch's membrane, and sub-RPE deposits in normal and AMD eyes. Invest Ophthalmol Vis Sci. 2002;43:2435-2441.

28. McLeod DS, Taomoto M, Otsuji T, et al. Quantifying changes in RPE and choroidal vasculature in eyes with age-related macular degeneration. Invest Ophthalmol Vis Sci. 2002;43:1986-1993.

29. Bron AJ, Tripathi RC, Tripathi BJ, eds. Wolff's Anatomy of the Eye and Orbit. 8th ed. London: Chapman and Hall; 1997. 
30. Mullins RF, Aptsiauri N, Hageman GS. Structure and composition of drusen associated with glomerulonephritis: implications for the role of complement activation in drusen biogenesis. Eye. 2001;15: 390-395.

31. Fryczkowski AW, Sherman MD. Scanning electron microscopy of human ocular vascular casts: the submacular choriocapillaris. Acta Anat (Basel). 1988;132:265-269.

32. Donders F. Beitrage zur patologishen anatomie des auges. Archiv Für Opbthalmologie. 1854;1:106-118.

33. Müller H. Anatomische beitrager zur ophthalmologie. Archiv Für Ophthalmologie. 1856;2:1-69.

34. Sarks SH. Drusen patterns predisposing to geographic atrophy of the retinal pigment epithelium. Aust J Ophthalmol. 1982;10:91-97.

35. Klaver CC, Assink JJ, van Leeuwen R, et al. Incidence and progression rates of age-related maculopathy: the Rotterdam Study. Invest Ophthalmol Vis Sci. 2001;42:2237-2241.

36. van Leeuwen R, Klaver CC, Vingerling JR, Hofman A, de Jong PT. The risk and natural course of age-related maculopathy: follow-up at $61 / 2$ years in the Rotterdam study. Arch Ophtbalmol. 2003; $121: 519-526$

37. Hageman GS, Mullins RF. Molecular composition of drusen as related to substructural phenotype. Mol Vis. 1999;5:28.
38. Lois N, Owens SL, Coco R, et al. Fundus autofluorescence in patients with age-related macular degeneration and high risk of visual loss. Am J Opbthalmol. 2002;133:341-349.

39. Malek G, Li CM, Guidry C, Medeiros NE, Curcio CA. Apolipoprotein $\mathrm{B}$ in cholesterol-containing drusen and basal deposits of human eyes with age-related maculopathy. Am J Pathol. 2003;162: 413-425.

40. Spraul CW, Lang GE, Grossniklaus HE, Lang GK. Histologic and morphometric analysis of the choroid, Bruch's membrane, and retinal pigment epithelium in postmortem eyes with age-related macular degeneration and histologic examination of surgically excised choroidal neovascular membranes. Surv Opbthalmol. 1999;44(supp1):S10-S32.

41. Müller H. Untersuchungen über die glashaute des auges, insbesondere die glaslamele der choroidea und ihre senile veranderungen. Arch Opbthalmol. 1856;2:1-65.

42. Woon WH, Fitzke FW, Bird AC, Marshall J. Confocal imaging of the fundus using a scanning laser ophthalmoscope. Br J Ophthalmol. $1992 ; 76: 470-474$.

43. Guymer R, Luthert P, Bird A. Changes in Bruch's membrane and related structures with age. Prog Retin Eye Res. 1999;18:59-90. 